\title{
The Effect of Chitosan Capped Copper Nanoparticles on Angiogenesis in Cam Assay
}

\author{
Harmankaya $0 F^{1}$, Hamsho $A^{2}$, Goztepe $\mathbf{M}^{1}$, Baghirova $S^{1}$, Cirik $S^{3}$, Sirin $\mathbf{H}^{5}$, Alasad $\mathbf{M}^{2}$, Sari $\mathbf{B S}^{1}$, \\ Uckun ${ }^{1}$, Kocer $A^{6}$, Aksu $E^{4}$ and Farmanli $0^{7 *}$ \\ ${ }^{1}$ Kırlkkale University School of Medinice, Turkey \\ ${ }^{2}$ Bioengineering Faculty, Kirıkkale University, Turkey \\ ${ }^{3}$ Sakarya University School of Medicine, Turkey \\ ${ }^{4}$ Bezmialem University School of Medicine, Turkey \\ ${ }^{5}$ Gülhane Education and Research Hospital, School of Medicine, Turkey \\ ${ }^{6}$ Selcuk University Vocational School of Health Services, Turkey \\ ${ }^{7}$ Department of Pathology, Marmara University School of Medicine, Turkey \\ *Corresponding author: Farmanli Orkhan, Department of Pathology, Marmara University School of Medicine, Turkey
}

\section{ARTICLE INFO}

Received: 幽 May 06, 2021

Published: 慧 May 13, 2021

Citation: Harmankaya OF, Hamsho A, Goztepe M, Baghirova S, Farmanli O, et al., The Effect of Chitosan Capped Copper Nanoparticles on Angiogenesis in Cam Assay. Biomed J Sci \& Tech Res 35(5)2021. BJSTR. MS.ID.005755.

Keywords: Angiogenesis; Chorioallantoic Membrane; Cancer; Copper Nanoparticles; Chitosan

\section{SUMMARY}

Objective: Angiogenesis is the growth of blood vessels from the existing vasculature that occurs during embryonic development, wound healing, and the menstruation cycle. Chitosan is a natural cationic polysaccharide and one of the natural polymers and obtained by deacetylation of $\alpha$-chitin. Chitosan-based nanoparticles show anti-cancer, anti-bacterial, anti-angiogenesis, procoagulant behaviors and good biosafety. In this study, the effects of chitosan-capped copper nanoparticles on angiogenesis in the chick chorioallantoic membrane (CAM) model were investigated.

Methods: We applied $25 \mathrm{mM}$ concentrations of CuNPs solution to CAM of 15 eggs and $15 \mathrm{mM}$ concentration of CuNPs solution to CAM of 16 eggs on the 5th day. After then, we evaluated results macroscopically on the 7th day.

Result: In our study, we observed that CuNPs solutions caused a significant increase in angiogenesis when compared with the control group. There was a greater increase in CAM vascular area angiogenesis of the $15 \mathrm{mM}$ solution group compared to the $25 \mathrm{mM}$ solution group.

Conclusion: The study of Ribeiro and colleagues with insulin-containing chitosan nanoparticles and our study with chitosan-coated copper nanoparticles stimulated angiogenesis. Although the study of Dragostin et al. shows that chitosan nanoparticles have an anti-angiogenic effect. We assumed that this effect may be due to copper nanoparticles as a result of our literature review.

\section{Introduction}

Angiogenesis is the growth of blood vessels from the existing vasculature [1] that occurs during embryonic development, wound healing, and the menstruation cycle. Moreover, unregulated angiogenesis (upregulation or downregulation) is seen in pathological conditions, such as psoriasis, diabetic retinopathy, and cancer [2]. Chitosan is a natural cationic polysaccharide and one of the natural polymers and obtained by deacetylation of $\alpha$-chitin $[3,4]$. It has been included in studies that chitosan molecule is an effective transportation tool for reaching the related target with the pharmaceutical properties of various drugs [5]. Chitosan-based nanoparticles also have major effects such as anti-cancer, antibacterial, anti-angiogenesis. Moreover, it was also demonstrated 
that chitosan nanoparticles suppress lipopolysaccharide-induced inflammatory cascade, which is prominent in Crohn's disease and ulcerative colitis [6]. It is known in the literature that chitosan has a procoagulant effect. Nanotoxicity study of chitosan nanoparticles was done in animal models. As a result of these studies, chitosan nanoparticles showed good biosafety [7].

Copper enters the human body through water and food, is mainly carried by plasmic proteins such as ceruloplasmin albumin, and its metabolism is in the co-factor structure of many enzymes [8]. They possess a great application in different fields such as antimicrobial activity, antifungal activity, sensors, catalysts, cytotoxicity "anticancer" activity, antioxidant activity [9]. The mesodermal layers of the allantoic and chorion fuse to form the chorioallantoic membrane (CAM) throughout avian development. CAM is an effective model to determine the behavior of normal and pathologic tissues (as cultured cells or malignant tumors). Using of various drugs, antibodies or biomaterials which placed on its surface allows us to observe angiogenesis [10]. CAM assay is preferred model by virtue of some advantages including, high embryo survival rate, easy methodology, sterility is not required, low cost, reproducibility, reliability [11]. In this study, the effects of chitosan capped copper nanoparticles on angiogenesis in chick chorioallantoic membrane (CAM) model were investigated.

\section{Material and Methods}

\section{Material}

All chemicals were analytical grade and used as purchased without further purification. Chitosan (75-85\% deacetylated) the medium molecular weight chitosan is $190-310 \mathrm{kDa}$, Acetic acid (99.8-100.5\%, MW=60.05 g/mol), Amonia (25\%) were purchased from Iceland, SIGMA-ALDRICH Co., Ltd, Copper (II) nitrate trihydrate $\mathrm{Cu}\left(\mathrm{NO}_{3}\right)_{2} * 3 \mathrm{H}_{2} \mathrm{O}(99.0 \%, \mathrm{MW}=241.60 \mathrm{~g} / \mathrm{mol})$, Carbon disulfide $\mathrm{CS}_{2}$ (99.5\%, MW=76.14) were purchased Germany MERCK Co., Ltd, Sodium borohydride (96\%, MW=25 g/mol) was purchased from Switzerland, FLUKA CHMIKA Co., Ltd. All solutions were prepared using double distilled water.

\section{Synthesis of Dithiocarbamate Chitosan (DTCCS)}

DTCCS were prepared with minor revision according to the method defined by Muzzarelli et al. [12], A mixture of $4 \mathrm{~g}$ of chitosan and $0.025 \mathrm{~mol}$ of ammonia was stirred in ethanol 95\% (v / v) at room temperature for half an hour. A solution of carbon disulfide $\mathrm{CS}_{2}$ (2 $\left.\mathrm{mL}\right)$ was then added slowly dropwise to the solution. After stirring about 2 hours, the resulting mixture was filtered under vacuum with a filter paper and the remnant was washed by $100 \%$ of ethanol. After washed the polymer dried at $60^{\circ} \mathrm{C}$. Finally, DTCCS was obtained.

\section{Synthesis of DTCCS Capped Copper Nanoparticles}

The synthesis of copper nanoparticle was done by alteration of the procedure used by Dang et al. [13], Firstly, $0.1 \mathrm{gr}$ of dithiocarbamate chitosan DTCCS was dissolved in $100 \mathrm{ml}$ of $\% 1$ acetic acid, then the polymer solution was added dropwise to the solution of 0.105 gr copper nitrate $\left(\mathrm{Cu}\left(\mathrm{NO}_{3}\right)_{2} .3 \mathrm{H}_{2} \mathrm{O}\right)$ in $10 \mathrm{ml}$ distilled water, while the color of copper solution changed from blue to light blue color. After stirring at 10,000 rpm by a high-speed homogenizer for 15 minutes the $\mathrm{PH}$ of the solution was changed from $\mathrm{PH}=3$ to $\mathrm{PH}=6$ by $1 \mathrm{M}$ of $\mathrm{NaOH}$ solution, after adding $\mathrm{NaOH}$ the solation color changed to be dark blue. After stirring for about 30 minutes by high-speed homogenizer $10 \mathrm{ml}$ of $\mathrm{NaBH}_{4}$ was added to give a rapid brown coloration which turned to dark red after about 10 minutes of mixing. The solution was stirred for an additional 30 minutes to complete the reaction. after boiling the solution the copper nanoparticles were precipitated by centrifugation at $5,000 \mathrm{rpm}$ for 15 minutes. The particles were washed repeatedly to ensure purity. Finally, the $\mathrm{Cu}-\mathrm{NPs}$ were dried at $40^{\circ} \mathrm{C}$.

\section{Fertilized Chicken Egg Preparation}

For the experiment, we put 36 fertilized chicken eggs in an incubator at $37^{\circ} \mathrm{C}$ and $85-90 \%$ relative humidity. We kept it in the incubator for 5 days. Then, on the $5^{\text {th }}$ day, we pierced the eggshell in the place corresponding to the CAM of the chicken eggs. We applied the CuNPs solution on the CAM and covered the hole of each egg with paraffin tape. Then we put the eggs back in the incubator. We kept it in the incubator at suitable temperature and humidity for 48 hours after the application. At the end of the 48th hour, we evaluated the vascular development macroscopically on the CAM and photographed with a stereomicroscope attached to a digital camera.

\section{Application of the Solution to Fertilized Chicken Eggs}

On the $5^{\text {th }}$ day, we pierced the eggshell in the place corresponding to the CAM of the chicken eggs. Before the application, we photographed the CAMs of the eggs with a stereomicroscope connected to a digital camera. We applied $15 \mathrm{mM}$ and $25 \mathrm{mM}$ of CuNPs solution to each CAM surface. After the application, we closed the holes of each egg with paraffin tape. We grouped and numbered eggs with $15 \mathrm{mM}$ and $25 \mathrm{mM}$ CuNPs solution applied [14]. Then we kept it in the incubator for 48 hours at suitable temperature and humidity. On the 7th day, we removed the paraffin tape on the CAM. All eggs were observed macroscopically and photographed with a stereomicroscope connected to the digital camera. Using the Knighton et al. score, the vascularization increases in the photographs taken on the 5th and 7th days were scored by 3 observers [15].

\section{Result}

On the $5^{\text {th }}$ day we pierced the eggshell. We used 36 eggs for this study. We established 3 eggs died before the application of CuNPs solutions. Two of 33 eggs were used as controls. We applied $20 \mathrm{~mL}$ volume CuNP solution in different concentrations to the CAM of the remaining 31 eggs. We applied $25 \mathrm{mM}$ concentrations of CuNPs 
solution to CAM of 15 eggs and $15 \mathrm{mM}$ concentration of CuNPs solution to CAM of 16 eggs on the $5^{\text {th }}$ day.

On the $7^{\text {th }}$ day, CAMs of eggs were evaluated macroscopically by 3 researchers. We rated effects CuNPs solution on CAM vessel growth and angiogenesis (Figures 1-3). The treatment of CAM with $25 \mathrm{mM}$ CuNPs solutions caused a significant development in CAM vascular area of 7 eggs. And the treatment of CAM with $15 \mathrm{mM}$

Table 1: Macroscopic evaluation of the effect of CuNPs treatment on CAM.

\begin{tabular}{|c|c|c|c|c|c|}
\hline \multicolumn{6}{|c|}{ Efficacy } \\
\hline Group & & Ineffective & 1 & 2 & Total \\
\hline \multirow{2}{*}{ Control } & $\mathrm{n}$ & 0 & 1 & 0 & 1 \\
\hline & $\%$ & 0 & 100 & 0 & 100 \\
\hline \multirow{2}{*}{$15 \mathrm{mM}$} & $\mathrm{n}$ & 6 & 7 & 3 & 16 \\
\hline & $\%$ & 37,5 & 43,75 & 18,75 & 100 \\
\hline \multirow{2}{*}{$25 \mathrm{mM}$} & $\mathrm{n}$ & 7 & 5 & 2 & 14 \\
\hline & $\%$ & 50 & 35,7 & 14,3 & 100 \\
\hline \multirow{2}{*}{ Total } & $\mathrm{n}$ & 14 & 12 & 5 & 31 \\
\hline & $\%$ & 45,15 & 38,7 & 16,15 & 100 \\
\hline
\end{tabular}

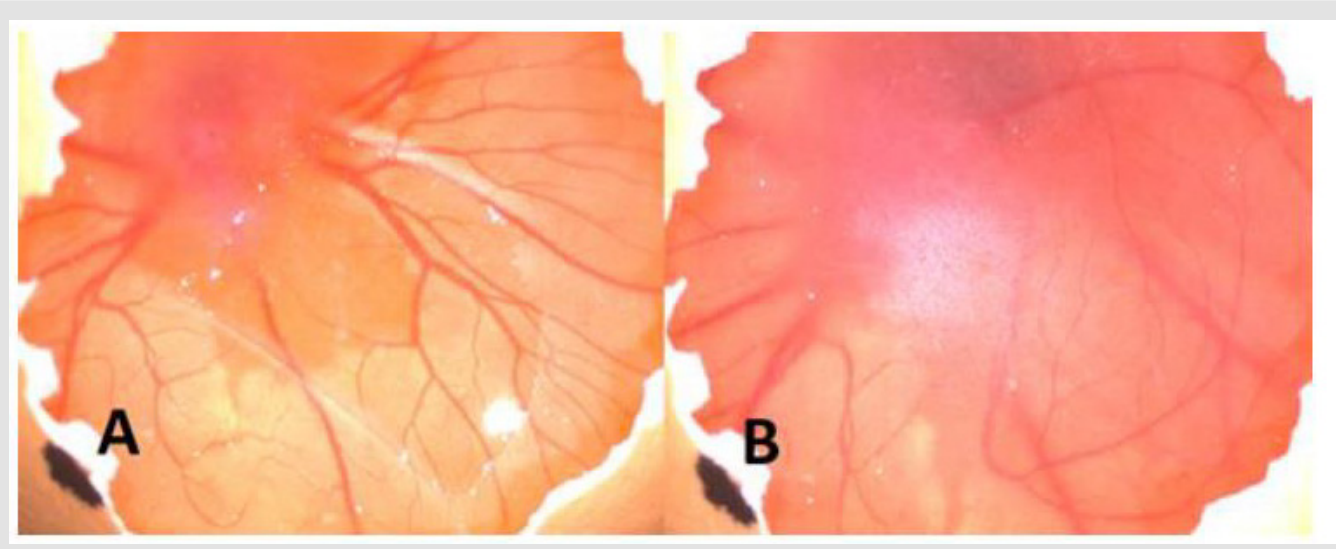

Figure 1: There is no any injection to A and B. As seen macroscopically there is no significant changes of angiogenesis status.

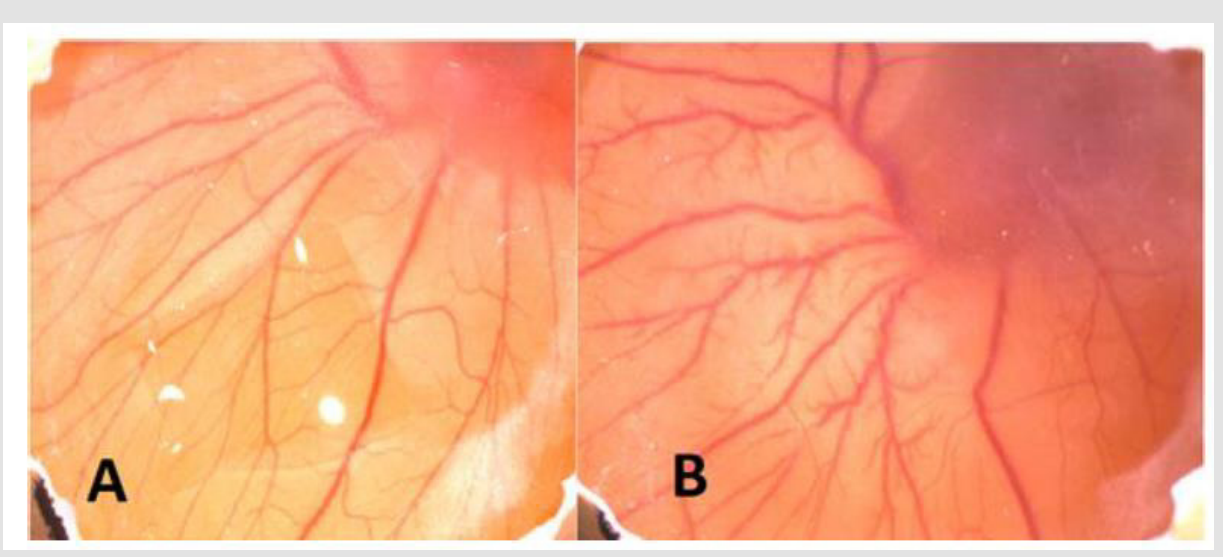

Figure 2: A. Before injection of CuNPs B. After injection of CuNPs. As seen macroscopically angiogenesis increased (+2) after injection. 


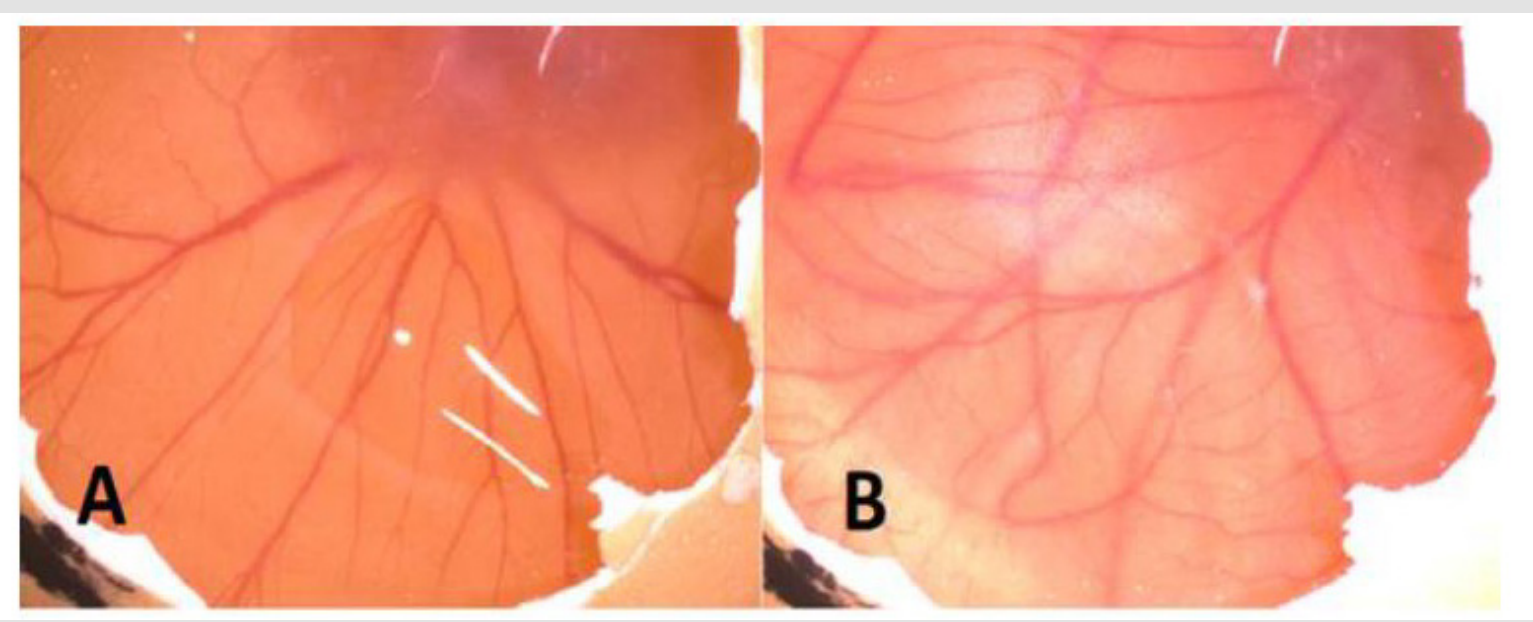

Figure 3: A. Before injection of CuNPs B. After injection of CuNPs. As seen macroscopically angiogenesis increased (+1) after injection.

\section{Discussion}

Angiogenesis is the process of generating new blood vessels and have essential roles in many pathological conditions [16]. Because of high sensitivity, being cheap and easy CAM model of chick embrios is mostly used as an in-vivo working model for angiogenesis [17]. Copper is a essential microelement required by animals and humans. Although the content of $\mathrm{Cu}$ in the human body is very low but it plays an important and multifunctional role as a cofactor of many enzymes [18]. Cu has essential role in angiogenesis as a stimulator of angiogenesis, vasculogenesis and endothelial cell migration [19]. $\mathrm{Cu}$ has been shown to stimulate angiogenesis in chick embryo chorioallantoic membrane (CAM) models [20]. Chitosan is the product of the complete or partial deacetylation of chitin. Chitosan is a widely used excipient for topical or oral drug administration. It is also now considered as a useful material for drug and gene delivery systems for parenteral administration [21]. Chitosan has been shown to inhibit angiogenesis in chick embryo chorioallantoic membrane (CAM) models [22].

Up to now, no study has been observed about angiogenic effect of Chitosan capped Copper nanoparticles except MroczekSosnowska et al. manuscript on chick chorioallantoic membrane assay in literature. Natalia Mroczek-Sosnowska et al. investigated the effect of copper nanoparticles on angiogenesis in CAM assay at the systemic and molecular level. As a result of the study, they proved that copper nanoparticles increase angiogenesis [18]. In a study by Yannan Li et al., the effect of copper on angiogenesis was examined using hydrogel scaffold. As a result of the study, they established that copper increases angiogenesis by increasing HIF$1 \alpha$ / VEGF expression [23]. As a result of our literature review about chitosan, we encountered a manuscript by Dragostin et al. They demonstrated the anti-angiogenic effect of chitosan nanoparticles in CAM assay [22]. In another study, Ribeiro and colleagues have shown that insulin-containing chitosan nanoparticles stimulate angiogenesis [24]. We thought this effect could be due to insulin. Similarly, in our study, chitosan coated copper nanoparticles stimulated angiogenesis. We assumed that this effect may be due to copper nanoparticle as a result of our literature review.

Consequently, Chitosan and copper nanoparticles are biomaterials in which experimental research are carried out in many areas such as cancer treatments, drug administrations, scaffolds. Therefore, it is essential to know the mechanism of action of these molecules. In our study, we also focused on the effects on angiogenesis which has an important role in many pathology processes. As a result of the study, we established that chitosan coated copper nanoparticles stimulate angiogenesis. Further studies are required.

\section{Conflict of Interest}

None.

\section{References}

1. Adam C Mirando (2017) Assessing the effects of threonyl-tRNA synthetase on angiogenesis-related responses. Methods 113: 132-138.

2. Tiziana Tonini, Francesca Rossi, Pier Paolo Claudio (2003) Molecular basis of angiogenesis and cancer. Oncogene 22: 6549-6556.

3. Antonella Arena (2017) Copper Oxide Chitosan Nanocomposite: Characterization and Application in Non-Enzymatic Hydrogen Peroxide Sensing, Sensors 17: 2198.

4. Alan Smith, Michael Perelman, Michael Hinchcliffe (2014) Chitosan, A promising safe and immune-enhancing adjuvant for intranasal vaccines. Human Vaccines \& Immunotherapeutics 10(3): 797-807.

5. Jianghua Li, Chao Cai, Jiarui Li, Jun Li, Jia Li, et al. (2018) Chitosan-Based Nanomaterials for Drug Delivery. Molecules 23(10): 2661.

6. Chun Y Wong, Hani Al-Salami, Crispin R Dass (2018) The role of chitosan on oral delivery of peptide-loaded nanoparticle formulation. Journal of Drug Targeting 26(7): 551-562. 
7. Sonin D, Evgeniia Pochkaeva, Sergei Zhuravskii, Viktor Postnov, Dmitry Korolev, et al. (2020) Biological Safety and Biodistribution of Chitosan Nanoparticles. Nanomaterials (Basel) 10(4).

8. Pohanka M (2019) Copper and copper nanoparticles toxicity and their impact on basic functions in the body. Bratisl Lek Listy 120(6): 397-409.

9. Mostafa F Al Hakkani (2020) Biogenic copper nanoparticles and their applications: A review. SN Applied Sciences 2: 505.

10. Cirligeriu Laura, Anca Maria Cimpean, Horia Calniceanu, Mircea Vladau, Simona Sarb, et al. (2018) Hyaluronic Acid/Bone Substitute Complex Implanted on Chick Embryo Chorioallantoic Membrane Induces Osteoblastic Differentiation and Angiogenesis, but not Inflammation 19(12): 4119.

11. Ribatti D (2014) The chick embryo chorioallantoic membrane as a model for tumor biology. Exp Cell Res 328: 314-324.

12. Muzzarelli RAA, Tanfani F, Mariotti S, Emanuelli M (1982) Preparation and characteristic properties of dithiocarbamate chitosan, a chelating polymer.

13. Dangm TMD, Le TTT, Fribourg-Blanc E, Dang MC (2011) The influence of solvents and surfactants on the preparation of copper nanoparticles by a chemical reduction method.

14. Kamble S, Bhimashankar Utage, Pratima Mogle, Rahul Kamble, Shrikant Hese, et al. (2016) Evaluation of Curcumin Capped Copper Nanoparticles as Possible Inhibitors of Human Breast Cancer Cells and Angiogenesis: a Comparative Study with Native Curcumin. AAPS Pharm Sci Tech 17: 1030-1041.

15. Knighton D, D Ausprunk, D Tapper, J Folkman (1977) Avascular and vascular phases of tumour growth in the chick embryo. Br J Cancer 35(3): 347-356.

ISSN: 2574-1241

DOI: 10.26717/BJSTR.2021.35.005755

Farmanli 0. Biomed J Sci \& Tech Res

(C) This work is licensed under Creative

Commons Attribution 4.0 License

Submission Link: https://biomedres.us/submit-manuscript.php
16. F Yesildal, FN Aydin, S Deveci, S Tekin, I Aydin, et al. (2015) Aspartame induces angiogenesis in vitro and in vivo models. Human \& Experimental Toxicology 34(3): 260-265.

17. Özgürtaş T (2009) Anjiyojenezde bir in-vivo model: civciv koriyoallantoik membran. Gülhane Tıp Dergisi 51: 67- 69.

18. Natalia Mroczek-Sosnowska, Ewa Sawosz, Krishna Prasad Vadalasetty, Monika Łukasiewicz, Jan Niemiec, et al. (2015) Nanoparticles of Copper Stimulate Angiogenesis at Systemic and Molecular Level Natalia. Int J Mol Sci 16: 4838-4849.

19. Finney L, Vogt S, Fukai T, Glesne D (2009) Copper and angiogenesis: Unravelling a relationship key to cancer progression. Clin Exp Pharmcol Phys 36: 88-89.

20. Gupte A, Mumper RJ (2009) Elevated copper and oxidative stress in cancer cells as a target for cancer treatment. Cancer Treat Rev 35: 32-46.

21. Dmitry Sonin, Evgeniia Pochkaeva, Sergei Zhuravskii, Viktor Postnov, Dmitry Korolev, et al. (2020) Biological Safety and Biodistribution of Chitosan Nanoparticles. Nanomaterials 10: 810.

22. Oana-Maria Dragostin (2020) Designing of Chitosan Derivatives Nanoparticles with Antiangiogenic Effect for Cancer Therapy, Nanomaterials 10: 698 .

23. Yannan Li, Tianzhen Xu, Zhuolong Tu, Wentong Dai, Yumeng Xue, et al (2020) Bioactive antibacterial silica-based nanocomposites hydrogel scaffolds with high angiogenesis for promoting diabetic wound healing and skin repair, Theranostics 10(11): 4929-4943.

24. Ribeiro MC, Viviane Lopes Rocha Correa, Francenya Kelley Lopes da Silva, Ariadine Amorim Casas , Angelica de Lima das Chagas, et al. (2020) Wound healing treatment using insulin within polymeric nanoparticles in the diabetes animal model. Eur J Pharm Sci 5: 105330.

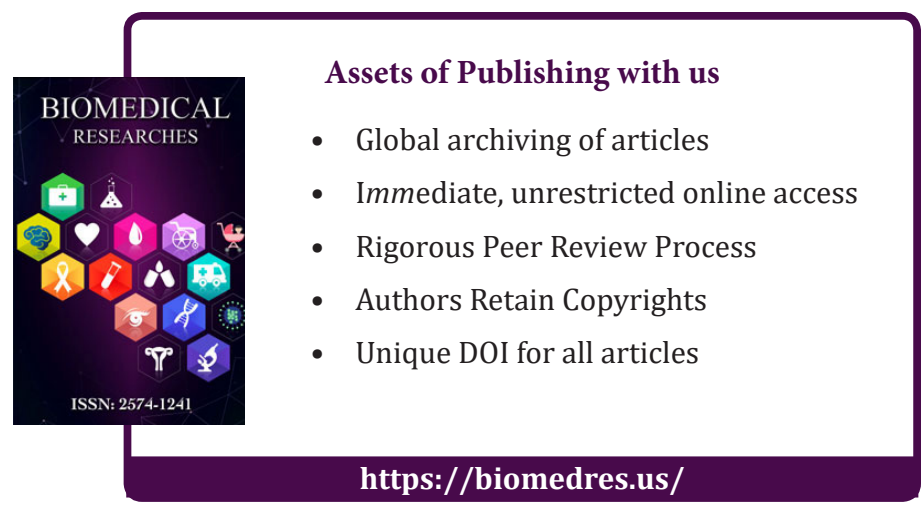

